\title{
EARLY RESULTS FROM THE ULTRA HEAVY COSMIC RAY EXPERIMENT
}

\author{
D. O'Sullivan, A. Thompson, J. Bosch, R. Keegan \\ Dublin Institute for Advanced Studies (DIAS), Ireland \\ Phone: +353-1-6621333, Fax: +353-1-6621477 \\ K.-P. Wenzel and F. Jansen \\ Space Science Dept of ESA, ESTEC, Noordwijk, The Netherlands \\ Phone:+31-1719-83573, Fax: +31-1719-84698 \\ C. Domingo \\ Universitat Autònoma de Barcelona, Spain \\ Phone:+34-3-581-1530, Fax: +34-3-581-2155
}

\begin{abstract}
Data extraction and analysis of the LDEF Ultra Heavy Cosmic Ray Experiment is continuing. Almost twice the pre LDEF world sample has been investigated and some details of the charge spectrum in the region from $Z \sim 70$ up to and including the actinides are presented. The early results indicate $r$ process enhancement over solar system source abundances.
\end{abstract}

\section{INTRODUCTION}

Prior to the launch of the LDEF, a number of major experiments designed to study ultra heavy nuclei in Earth orbit had already been completed and the Heavy Nucleus Experiment on HEAO- $3^{1}$ along with the Bristol experiment on board Ariel$\mathrm{VI}^{2}$ provided most of the data available at that time.

Two important requirements for a successful experiment in the study of charge spectra are good charge resolution and a sufficiently large sample of data. The DublinESTEC experiment has collected almost fifteen times the combined (HEAO-3 + ArielVI) data set and there are indications that the charge resolution is at least as good, if not better than planned. To put things further in perspective, the total number of actinides $(Z \geq 87)$ observed in these earlier experiments combined was 3 , whereas preliminary results for the Dublin-ESTEC experiment indicate a total of $\sim 70 \pm 15$ actinides in the sample obtained. 


\section{Astrophysical Significance of Ultra Heavy Nuclei}

Ultra heavy nuclei are synthesised by the neutron capture process. This reaction is characterised by two distinct types which depend on the rate at which capture takes place, namely the slow $(\mathrm{s})$ and rapid $(\mathrm{r})$ processes. The contribution of each process to the abundance of nuclei with $Z>30$ varies widely from element to element. Accordingly, a detailed knowledge of relative abundances can provide evidence for either $\mathbf{s}$ or $\mathbf{r}$ process domination in a given charge region. The key to solving some problems associated with cosmic ray origin and acceleration is the fact that the $\mathrm{s}$ and $\mathrm{r}$ processes occur in quite different astrophysical environments so that the measured abundance should reflect different nucleosynthesis histories and provide information on the source of ultra heavy nuclei. The r-process is usually associated with supernova explosions, whereas the s-process is believed to take place in normal stars at an advanced stage of evolution.

To extract the interesting astrophysical implications from data measured in Earth orbit they are usually compared with values derived from models based on particular types of source abundances which have been corrected for fractionation and propagation through the Galaxy. The effect of the first ionisation potential (FIP) ${ }^{3}$ of each element in determining its likelihood of being ionised and accelerated as part of the galactic cosmic ray flux is now well established and is also taken into account in these models. The HEAO-3 and Ariel-VI groups used the standard leaky box model of propagation along with the rigidity dependent path length distribution of Ormes and Protheroe and the semiempirical fragmentation cross sections of Silberberg and Tsao ${ }^{5}$ which are assumed to be independent of energy above $\sim 1 \mathrm{GeV} / \mathrm{N}$. The main conclusions drawn by the HEAO-3 and Ariel-VI groups were that (i) for the $33 \leq Z \leq 60$ charge region the observed abundances agree well with solar system source material with FIP fractionation with a slight enrichment of $r$-process material compared to the accepted solar system mixture, (ii) $\operatorname{Pt}(Z=78)$ and its secondaries are best fitted by a predominantly r-process source with FIP fractionation. It may seem strange that cosmic rays should be richer in $r$-process material at the upper end of the periodic table than in the region below $N d(Z=60)$ but there are r-process conditions which synthesise only nuclei with $Z>60$.

\section{Experiment Design}

Details of the Dublin-ESTEC experiment designed to investigate cosmic ray nuclei with $Z>65$ are given in several papers. A total of 192 detector stacks, comprised mainly of lexan polycarbonate, were mounted in sets of four within cylindrical eccofoam moulds which were then inserted into 48 separate aluminium pressure vessels ( 1 atm of dry air). Following recovery of the LDEF after 69 months in space the gas pressure in all cylinders was checked and it was found that no leakage had occurred. 
The experiment thermal design was extremely successful and resulted in an average detector stack temperature below $0^{\circ} \mathrm{C}$ with a mean maximum spread of $\pm 10^{\circ} \mathrm{C}$. The corresponding charge shifts were $\pm 0.8 e$ for uranium and $\pm 0.6 e$ for the $\mathrm{Pt}-\mathrm{Pb}$ region.

A very fortuitous aspect of the life time of the Dublin-ESTEC experiment was the close parallel with the life time of the facility at the Berkeley Bevalac for accelerating ultra heavy nuclei such as uranium and gold. The facility started in time for preflight calibration before launch in 1984 and survived long enough to allow post flight calibration of the detectors before it closed down in February 1993, much to the regret of the cosmic ray community. Analysis of the pre and post flight data has shown that the strict control of detector environment throughout the mission has resulted in no observable degradation of latent image ${ }^{6}$.

\section{Locating and Identifying Ultra Heavy Cosmic Ray Nuclei in the Lexan Polycarbonate Stacks}

Locating the ultra heavy nuclei is straightforward. Because of the orbital inclination only high energy $(E \geq 2 \mathrm{GeV} / N)$ particles reach the detectors. The ionisation threshold for lexan is such that at these energies only particles with $Z \geq 65$ register and there is no significant background from lighter nuclei (a major consideration when you spend almost 5 years in space!) Approximately 2500 particles with $Z>65$ have been located.

Following carefully controlled etching of 20 plates from the top of each stack and a similar number from the bottom, a set of the 40 etch rate values $V_{t}$ were determined for each ultra heavy candidate. Charge identification is based on measurement of (i) G, the fractional etch rate gradient; $G=\frac{1}{S} \frac{d S}{d X}$ where $S=\frac{V_{t}}{V_{g}}, V_{g}$ is the bulk etch rate and $\mathrm{X}$ is the path length and (ii) the effective reduced etch rate $S_{\text {eff }}$. S is related to a restricted energy loss function through $S=g(R E L)^{h}$ where $\mathrm{g}$ and h are determined from our $\mathrm{U}(\sim 950 \mathrm{MeV} / \mathrm{N})$ and $\mathrm{Au}(\sim 1150 \mathrm{MeV} / N)$ calibrations at the Bevalac. Typical $\mathrm{S}$ versus path length plots are shown in references 7 and 8 .

\section{The Preliminary Charge Spectrum Results and Discussion}

The preliminary charge spectrum, based on approximately $15 \%(\sim 450$ events) of the total sample, is shown in Fig 1. The presence of nuclei beyond the actinide gap is significant and in view of the modest charge spread $(\sim 1 e)$ expected from statistical errors and temperature excursions we are confident even at this early stage that the Dublin-ESTEC experiment is capable of providing the first statistically significant sample of cosmic ray nuclei with $Z>87$ with good charge resolution. How 'good' this resolution will eventually be remains to be seen, but at present we have no reason to believe that it will not be well within the design target of $<1.5 e$, and probably better for a sample of events with appropriate path lengths and geometry. 


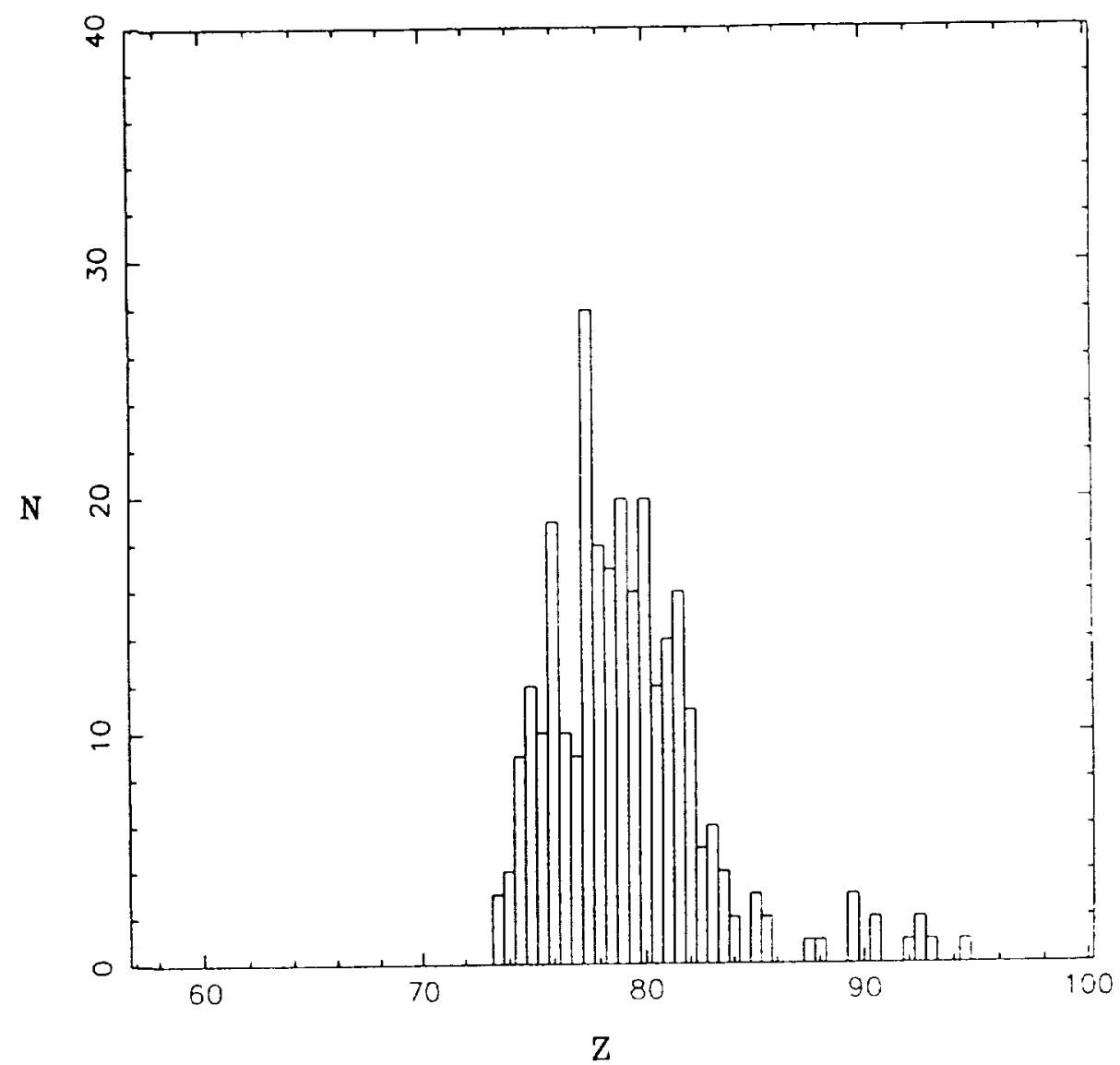

Figure 1: A preliminary charge spectrum for nuclei with $Z>70$ based on $\approx 450$ nuclei $(\approx 15 \%$ of total sample) from the DublinESTEC experiment.

Selection from stacks with the smallest temperature excursions will also help and the possibility of obtaining abundance ratios for charge groups (and even a $\mathrm{Th} / \mathrm{U}$ value) in this region looks promising. The ratio of $(Z \geq 88) /(74 \leq Z \leq 87)$ from Fig 1 is $0.031 \pm 0.009$ compared with $0.025 \pm 0.015$ for the combined HEAO-3+Ariel-VI data. It should be emphasised however that this early value from the Dublin-ESTEC experiment should be taken as a lower value since no correction has yet been applied for any differences that may exist in the fragmentation cross-section of actinides as opposed to those for nuclei in the $74 \leq Z \leq 87$ charge region ${ }^{9}$. The relative significance of fission and electromagnetic interactions at high energies for both of these groups of nuclei will be the subject of future investigations. Our initial method of scanning, while efficient at locating those nuclei which survive $\frac{2}{3}$ of their trajectory through the stacks, does not pick up those which interact and lose sufficient charge to fall below threshold $(Z \sim 65)$, as would occur in fission for example. We would expect this effect to be highest for the actinide elements. We hope to tackle this problem shortly. Further 
down the charge scale we note that the spectrum peaks around $Z=77.5$, indicating the presence of predominantly $r$-process nuclei such as osmium and platinum, while $\mathrm{Pb}$ (predominantly s-process) is not very dominant.

Within another year we hope to have completed measurements on a total of 1000 ultra heavy nuclei and will have had the opportunity to consider any systematic effects which may exist before trying any serious deconvolution of the spectral peaks. The overall situation at present certainly gives good reason for optimism.

\section{REFERENCES}

1. W.R. Binns, T.L. Garrard, P.S. Gibner, M.H. Israel, M.P. Kertzmann, J. Klarmann, B.J. Newport, E.C. Stone and C.J. Waddington, The Astrophysical Journal, (1989), 346, 997-1009

2. P.H. Fowler, R.N.F. Walker, M.R.W. Masheder, R.T. Moses, A. Worley and A.M. Gay, The Astrophysical Journal, (1987), 314, 739-746

3. J.R. Letaw, R. Silberberg and C.H. Tsao, Ap. J., (1984), 279, 144

4. J.F. Ormes and R.J. Protheroe, Ap. J., (1983), 272, 756

5. R. Silberberg and C.H. Tsao, Ap. J. Suppl., (1973), 25, 315

6. A. Thompson, D. O'Sullivan, K-P Wenzel, J. Bosch, R. Keegan, C. Domingo, J. Daly and A. Smit, Proc 22nd ICRC, (1991), 2, 543, (Dublin)

7. D. O'Sullivan, A. Thompson, J. Bosch, R. Keegan, K-P Wenzel, F. Jansen, C. Domingo, Adv. Sp. Res., (1993), to be published.

8. A. Thompson, D. O'Sullivan, J. Bosch, R. Keegan, K-P Wenzel, F. Jansen and C. Domingo, Proc Second LDEF Post-Retrieval Symposium, (1992), 1, 261, (San Diego)

9. L.Y. Geer, J. Klarmann, B.S. Nilsen, C.J. Waddington, W.R. Binns, J.R. Cummings and T.L. Garrard, Proc 29rd ICRC, (1993), 2, 191, (Calgary) 
